\title{
Head and Neck Fluids and Secretions
}

National Cancer Institute

\section{Source}

National Cancer Institute. Head and Neck Fluids and Secretions. NCI Thesaurus. Code C13418.

Secretions and fluids from the area of the body generally construed to comprise base of skull and facial bones, sinuses, orbits, salivary glands, oral cavity, oropharynx, larynx, thyroid, facial and neck musculature and lymph nodes draining these areas. 